\title{
Correction: Niclosamide and its analogs are potent inhibitors of Wnt/ $\beta$-catenin, mTOR and STAT3 signaling in ovarian cancer
}

\author{
Rebecca C. Arend ${ }^{1}$, Angelina I. Londoño-Joshi ${ }^{2}$, Abhishek Gangrade ${ }^{2}$, Ashwini A. \\ Katre $^{2}$, Chandrika Kurpad ${ }^{2}$, Yonghe Li ${ }^{3}$, Rajeev S. Samant ${ }^{4}$, Pui-Kai Li ${ }^{5}$, Charles \\ N. Landen ${ }^{6}$, Eddy S. Yang ${ }^{2}$, Bertha Hidalgo, Ronald D. Alvarez ${ }^{1}$, John Michael \\ Straughn $^{1}$, Andres Forero ${ }^{8}$ and Donald J. Buchsbaum ${ }^{2}$ \\ ${ }^{1}$ University of Alabama at Birmingham, Department of Obstetrics and Gynecology, Division of Gynecologic Oncology, \\ Birmingham, AL, USA \\ ${ }^{2}$ University of Alabama at Birmingham, Department of Radiation Oncology, Birmingham, AL, USA \\ ${ }^{3}$ Southern Research Institute, Department of Oncology, Birmingham, AL, USA \\ ${ }^{4}$ University of Alabama at Birmingham, Department of Pathology, Division of Molecular \& Cellular Pathology, Birmingham, \\ $\mathrm{AL}$, USA \\ ${ }^{5}$ Ohio State University, Department of Medicinal Chemistry and Pharmacognosy, Columbus, OH, USA \\ ${ }^{6}$ University of Virginia, Department of Oncology, Division of Gynecologic Oncology, Charlottesville, VA, USA \\ ${ }^{7}$ University of Alabama at Birmingham, Department of Epidemiology, Birmingham, AL, USA \\ ${ }^{8}$ University of Alabama at Birmingham, Department of Medicine, Division of Hematology \& Oncology, Birmingham, AL, USA \\ Published: April 10, 2018
}

Copyright: Arend et al. This is an open-access article distributed under the terms of the Creative Commons Attribution License 3.0 (CC BY 3.0), which permits unrestricted use, distribution, and reproduction in any medium, provided the original author and source are credited.

This article has been corrected: The correct Funding information is given below:

\section{FUNDING}

NIH grant support in the Comprehensive Flow Cytometry Core (CFCC) (P30 AR48311), Dr. Londono is funded by a NIH Research Training Program in Basic and Translation Oncology T32 Grant (T32CA183926). Dr. Arend is supported by the Foundation for Women's Cancer (WeRoc/OChO Ovarian Cancer Research Grant), Norma Livingston Foundation, AAOGF/ ABOG Career Development Award, and ACS-IRG (IRG-60-001-53-IRG). Dr. Hidalgo is supported by the NHLBI Diversity Supplement (HHSN268201300001) and a grant from the Robert Wood Johnson Foundation, New Connections Program.

Original article: Oncotarget. 2016; 7:86803-86815. https://doi.org/10.18632/oncotarget.13466 